\title{
Densidade de estocagem e salinidade da água na larvicultura do pacamã
}

\author{
Ronald Kennedy Luz ${ }^{(1)}$ e José Cláudio Epaminondas dos Santos ${ }^{(2)}$
}

\begin{abstract}
(1)Pontifícia Universidade Católica de Minas Gerais, Departamento de Zoologia de Vertebrados, Prédio 41, Avenida Dom Gaspar, no 500, Coração Eucarístico, CEP 30535-610 Belo Horizonte, MG. E-mail: luzrk@yahoo.com ${ }^{(2)}$ Companhia de Desenvolvimento dos Vales do São Francisco e do Parnaíba, Estação de Hidrobiologia e Piscicultura de Três Marias, Caixa Postal 11, CEP 39205-000 Três Marias, MG. E-mail: jose.claudio@codevasf.gov.br
\end{abstract}

\begin{abstract}
Resumo - O objetivo deste trabalho foi avaliar o efeito da densidade de estocagem e da salinidade da água, na larvicultura do pacamã Lophiosilurus alexandri, alimentado com náuplios de Artemia sp. Foram utilizadas três densidades de estocagem: 20, 40 e 60 larvas L $\mathrm{L}^{-1}\left(\mathrm{D}_{20}, \mathrm{D}_{40}\right.$ e $\mathrm{D}_{60}$, respectivamente). Para cada densidade testaram-se três índices de salinidade da água: água doce $\left(\mathrm{S}_{0}\right), 2 \%_{0}\left(\mathrm{~S}_{2}\right) \mathrm{e} 4 \%$ o $\left(\mathrm{S}_{4}\right)$, em delineamento experimental inteiramente ao acaso, em esquema fatorial $3 \times 3$, com três repetições. Foram analisados: crescimento, sobrevivência, mortalidade, canibalismo, produtividade e níveis de amônia não ionizada. As concentrações de amônia não ionizada foram maiores nas densidades $D_{40}$ e $D_{60}$. Aos dez dias de alimentação, o peso foi afetado negativamente em $D_{40}$ e $D_{60}$ associado à maior salinidade da água $\left(S_{4}\right)$. A maior sobrevivência foi obtida no tratamento $D_{20} S_{2}$, enquanto os menores valores foram registrados em relação à salinidade $\mathrm{S}_{4}$, independentemente da densidade testada. Foram verificadas baixas taxas de mortalidade e ausência de canibalismo. A produção de juvenis também foi influenciada pelos fatores avaliados. Larvas de L. alexandri podem ser criadas, durante os dez primeiros dias de alimentação exógena, em água doce ou a $2 \%$ com maior produção de juvenis na densidade $\mathrm{D}_{60}$.
\end{abstract}

Termos para indexação: Artemia, Lophiosilurus alexandri, criação intensiva, produção de juvenis.

\section{Stocking density and water salinity on pacamã larviculture}

\begin{abstract}
The objective of this work was to evaluate the effect of stocking density and water salinity levels on Lophiosilurus alexandri (pacamã) larvae rearing fed with Artemia sp. nauplii. Three stocking densities were used: 20,40 and 60 larvae $\mathrm{L}^{-1}\left(\mathrm{D}_{20}, \mathrm{D}_{40}\right.$ and $\mathrm{D}_{60}$, respectively). For each density, three different levels of salinity were tested: freshwater $\left(\mathrm{S}_{0}\right), 2 \%$ o $\left(\mathrm{S}_{2}\right)$ and $4 \%$ o $\left(\mathrm{S}_{4}\right)$, in a completely randomized design, in a $3 \times 3$ factorial scheme, with three replications. The growth, survival, mortality, cannibalism, production and nonionized ammonia levels were evaluated. The nonionized ammonia levels were higher at $\mathrm{D}_{40}$ and $\mathrm{D}_{60}$. At 10 days of feeding, the weight was affected negatively in $\mathrm{D}_{40}$ and $\mathrm{D}_{60}$ associated to higher water salinity $\left(\mathrm{S}_{4}\right)$. The higher survival was verified in $\mathrm{D}_{20} \mathrm{~S}_{2}$ treatment, while smaller values were found in $\mathrm{S}_{4}$, regardless of the density used. Small mortality rate and no cannibalism were observed. The juvenile final production was also influenced by the evaluated variables. Larvae of $L$. alexandri can be reared, during the first ten days of exogenous feeding, in freshwater or slightly saline water $(2 \%)$ with higher juvenile production at density $\mathrm{D}_{60}$.
\end{abstract}

Index terms: Artemia sp., Lophiosilurus alexandri, intensive husbandry, juvenile production.

\section{Introdução}

O pacamã Lophiosilurus alexandri é um peixe da família Pseudopimelodidae (Ordem Siluriformes), nativo da bacia do rio São Francisco (Shibata, 2003), que apresenta alto valor de mercado por sua carne sem espinhos intramusculares e pelo sabor apreciado pelo consumidor. É uma espécie que apresenta hábito alimentar carnívoro, com comportamento sedentário, desova parcelada e preferência por ambientes lênticos em regiões de fundo de areia ou de pedras (Travassos, 1959). A literatura sobre a criação de pacamã é escassa. López \& Sampaio (2000) reportam que larvas dessa espécie, criadas em diferentes densidades de estocagem e alimentadas com zooplâncton, apresentam canibalismo acentuado.

A densidade de estocagem é um dos fatores que tem merecido atenção especial na larvicultura de peixes, por afetar a sobrevivência (Luz \& Zaniboni Filho, 2002; Campagnolo \& Nuñer, 2006), o crescimento 
(Baskerville-Bridges \& Kling, 2000; Bolasina et al., 2006) e o comportamento das larvas (Kestemont et al., 2003; Andrade et al., 2004). O uso de quantidades reduzidas de animais leva à subtilização do espaço disponível para a criação, e densidades muito elevadas também podem ser prejudiciais, em razão da maior quantidade de alimento utilizada e sua conseqüente degradação, e à maior excreção de resíduos nitrogenados pelos peixes, com prejuízos na qualidade da água (Jobling, 1994).

De acordo com Zaniboni Filho (2000), a larvicultura intensiva de peixes neotropicais pode ser realizada com densidades de estocagem entre 15 e 30 larvas L $^{-1}$. No entanto, para Hoplias lacerdae, espécie neotropical carnívora, a larvicultura intensiva pode ser realizada com densidade de até 90 larvas $\mathrm{L}^{-1}$, sem afetar o desempenho dos animais e a produção (Luz \& Portella, 2005). Segundo Lee \& Ostrowski (2001), as densidades de estocagem de espécies marinhas criadas nos Estados Unidos variam entre 20 e 40 larvas $\mathrm{L}^{-1}$. Para outros peixes marinhos, Shields (2001) relata a utilização de 50-150 larvas $\mathrm{L}^{-1}$ de Dicentrarchus labrax e Sparus aurata, 30 larvas $\mathrm{L}^{-1}$ de Scophthalmus maximus, e 10 larvas $\mathrm{L}^{-1} \mathrm{de}$ Hippoglossus hippoglossus.

Além da densidade, a qualidade do alimento a ser fornecido também é fundamental para o sucesso da larvicultura. Náuplios de Artemia sp. podem ser oferecidos às larvas de peixes, desde a primeira alimentação, com boa aceitação entre as várias espécies de água doce (Luz \& Zaniboni Filho, 2001; Jomori et al., 2003; Luz \& Portella, 2005; Feiden et al., 2006). Porém, por ser um organismo marinho, os náuplios tem seu tempo de vida limitado em água doce (Beux \& Zaniboni Filho, 2006).

Uma alternativa para o melhor aproveitamento dos náuplios de Artemia sp., pelas larvas de peixes de água doce, é a realização da larvicultura em águas ligeiramente salinizadas, manejo que tem apresentado bons resultados durante a larvicultura de algumas espécies (Luz \& Portella, 2002; Weingartner \& Zaniboni Filho, 2004; Campagnolo \& Nuñer, 2006). A água salinizada aumenta o tempo de vida dos náuplios de Artemia sp. (Beux \& Zaniboni Filho, 2006), e pode: ter implicações no crescimento pelo aumento ou diminuição da ingesta de alimento; reduzir os níveis de cortisol no plasma sanguíneo; alterar o comportamento animal, em relação à atividade antecipatória à alimentação (Luz et al., 2008); e ser utilizada na prevenção e controle de Ichthyophthirius multifiliis
(Garcia et al., 2007), parasita que tem sido comum em peixes de água doce.

O objetivo deste trabalho foi avaliar o efeito da densidade de estocagem e da salinidade da água, na larvicultura do pacamã L. alexandri alimentado com náuplios de Artemia sp.

\section{Material e Métodos}

O experimento foi realizado na Estação de Hidrobiologia e Piscicultura de Três Marias, da Companhia de Desenvolvimento dos Vales do São Francisco e do Parnaíba (Codevasf), em Três Marias, MG, em janeiro de 2007, durante os primeiros dez dias de alimentação exógena dos animais.

Larvas recém-eclodidas de L. alexandri foram mantidas em uma canaleta de $0,43 \mathrm{~m}^{2}$, em sistema de fluxo contínuo $\left(100 \mathrm{~mL} \mathrm{~min}^{-1}\right)$ e a $25,6 \pm 0,7^{\circ} \mathrm{C}$. Com oito dias pós-eclosão, larvas com 15,5 $\pm 0,7 \mathrm{~mm} \mathrm{e} 25,7 \pm 3,7 \mathrm{mg}$ foram estocadas em 27 aquários com $2 \mathrm{~L}$ cada um, sob aeração constante (oxigênio dissolvido $>5 \mathrm{mg} \mathrm{L}^{-1}$ ). Durante o experimento, a temperatura da água apresentou valores médios de $25,8 \pm 0,5$ e $26 \pm 0,4^{\circ} \mathrm{C}$ às 9 e $17 \mathrm{~h}$, respectivamente.

Foram utilizadas três densidades de estocagem: 20, 40 e 60 larvas L $\mathrm{L}^{-1}\left(\mathrm{D}_{20}, \mathrm{D}_{40}\right.$ e $\mathrm{D}_{60}$, respectivamente). Para cada densidade, utilizaram-se diferentes índices de salinidade da água: água doce, $2 \%$ e $4 \%$ o de salinidade $\left(\mathrm{S}_{0}, \mathrm{~S}_{2}\right.$ e $\mathrm{S}_{4}$, respectivamente). Utilizou-se um delineamento experimental inteiramente ao acaso, em esquema fatorial $3 \times 3$, três densidades de estocagem e três índices de salinidade, com três repetições de cada tratamento.

Náuplios de Artemia sp., eclodidos em água salinizada a $30 \%$, foram separados dos cistos e mantidos à salinidade de $10 \%$ o para serem fornecidos às larvas, nas quantidades diárias de 1.000 náuplios por larva, do 1ㅇ ao 5 o dia, e de 1.500 náuplios por larva, do 6o ao 10 o dia de alimentação, divididas em três refeições: às $9,13 \mathrm{e} 17 \mathrm{~h}$. Estas quantidades foram previamente estabelecidas para essa espécie na Estação de Piscicultura.

Diariamente, antes da primeira alimentação, os aquários foram sifonados para a retirada das sobras de alimento, e a água foi renovada em aproximadamente $50 \%$ do seu volume total. Nessa ocasião, as larvas mortas foram quantificadas. A concentração de amônia não ionizada também foi verificada, no 60 dia de experimento, pelo método de Koroleff (1976), em todas as unidades experimentais. 
Os diferentes meios salinizados foram previamente preparados em caixas de $500 \mathrm{~L}$ (soluções estoques), e a condutividade e o $\mathrm{pH}$ foram aferidos com o auxílio do aparelho Horiba U10. Foram registrados valores de 7,15, 7,19 e 7,21 para o $\mathrm{pH}$ e de 0,05, 3,78 e 6,54 para a condutividade $\left(\mathrm{mS} \mathrm{cm}^{-1}\right)$ nas salinidades $\mathrm{S}_{0}, \mathrm{~S}_{2}$ e $\mathrm{S}_{4}$, respectivamente. Para a salinização da água utilizou-se sal grosso não iodado.

Para se manter a proporcionalidade nos tratamentos, aos cinco dias de alimentação, foram coletados $10 \%$ de animais de cada réplica, considerando-se o número de indivíduos estocados inicialmente em cada densidade. Aos dez dias de experimento, foram amostrados mais $10 \%$ de indivíduos de cada réplica, correspondentes às diferentes densidades. Os animais foram sacrificados em gelo e fixados em formol a 10\%, para análises posteriores de comprimento total e peso. Com os resultados médios de peso inicial $\left(\mathrm{Pt}_{\mathrm{i}}\right)$ e peso final $\left(\mathrm{Pt}_{\mathrm{f}}\right)$ de cada réplica, foi calculada a taxa de crescimento específico pela equação: TCE $(\%$ por dia $)=100\left(\ln \mathrm{Pt}_{\mathrm{f}}-\ln \mathrm{Pt}_{\mathrm{i}}\right) / \Delta \mathrm{t}$, considerando-se $\Delta \mathrm{t}$ a duração em dias entre amostragens.

Aos dez dias de alimentação, foi avaliada a sobrevivência. A taxa de mortalidade foi calculada, tendo-se considerado os indivíduos retirados mortos e intactos. A taxa de canibalismo foi calculada pela diferença entre a taxa de sobrevivência e a mortalidade, ou seja, animais que desapareceram dos aquários e animais com sinais aparentes de agressão. Com o número de indivíduos vivos e seus respectivos pesos, calculou-se também a biomassa total produzida por aquário.
Valores percentuais de sobrevivência, mortalidade e taxa de crescimento específico foram transformados em $\operatorname{arc~sen}(\mathrm{x} / 100)^{0,5}$, para as análises estatísticas. Os dados foram submetidos à ANOVA fatorial paramétrica pelo teste $\mathrm{F}$, e as médias foram comparadas pelo teste de Tukey, a 5\% de probabilidade, pelo programa Statistica 7.0 (StatSoft, 2004).

\section{Resultados e Discussão}

As concentrações de amônia não ionizada, registradas na larvicultura de L. alexandri, foram influenciadas somente pela densidade de estocagem $(\mathrm{p}<0,05)$, com maior valor na $\mathrm{D}_{40}$, intermediário na $\mathrm{D}_{60}$ e inferior na $\mathrm{D}_{20}$ (Tabela 1). Apesar de a amônia não ionizada poder ser influenciada pela temperatura da água e pH (Emerson et al., 1975) e pela temperatura e salinidade (Bower \& Bidwell, 1978), no presente trabalho, a densidade de estocagem exerceu maior influência nas concentrações de amônia não ionizada que as diferenças no $\mathrm{pH}$ e na salinidade da água. $\mathrm{O}$ aumento nos níveis de amônia, com o aumento da densidade, também foi verificado na larvicultura de Gadus morhua (Baskerville-Bridges \& Kling, 2000), H. lacerdae (Luz \& Portella, 2005) e Pseudoplatystoma corruscans (Campagnolo \& Nuñer, 2006). Para Luz \& Portella (2005), o aumento nos níveis de amônia, em altas densidades, pode estar relacionado à maior excreção dos animais, como também à morte rápida dos náuplios de Artemia sp. em água doce. Segundo Beux \& Zaniboni Filho (2006), os náuplios de

Tabela 1. Análise de variância e médias ( \pm desvio-padrão) de amônia não ionizada, no sexto dia de larvicultura; comprimento total (CT), peso (P) e taxa de crescimento específico (TCE), aos cinco e dez dias de alimentação; sobrevivência (S), mortalidade (M), número de indivíduos e biomassa total de juvenis de Lophiosilurus alexandri, aos dez dias de alimentação, submetidos a três densidades de estocagem e três índices de salinidade da água ${ }^{(1)}$.

\begin{tabular}{|c|c|c|c|c|c|c|c|c|c|c|c|}
\hline \multirow[t]{2}{*}{ Fonte de variação } & \multicolumn{11}{|c|}{ Valores de F } \\
\hline & $\begin{array}{c}\text { Amônia não } \\
\text { ionizada } \\
\left(\mu \mathrm{g} \mathrm{L}^{-1}\right)\end{array}$ & $\begin{array}{c}\text { CT (mm) } \\
\text { (5 dias) }\end{array}$ & $\begin{array}{l}\text { CT (mm) } \\
\text { (10 dias) }\end{array}$ & $\begin{array}{l}\mathrm{P}(\mathrm{mg}) \\
\text { (5 dias) }\end{array}$ & $\begin{array}{l}\mathrm{P}(\mathrm{mg}) \\
\text { (10 dias) }\end{array}$ & $\begin{array}{c}\mathrm{TCE}^{(2)} \\
\text { (\% por dia) } \\
(5 \text { dias })\end{array}$ & $\begin{array}{c}\mathrm{TCE}^{(2)} \\
\text { (\% por dia) } \\
(10 \text { dias })\end{array}$ & $\mathrm{S}(\%)^{(2)}$ & $\mathrm{M}(\%)^{(2)}$ & $\begin{array}{c}\mathrm{N}^{\mathrm{o}} \mathrm{de} \\
\text { indivíduos }\end{array}$ & $\begin{array}{l}\text { Biomassa } \\
\text { total (mg) }\end{array}$ \\
\hline Densidade (D) & $4,47^{*}$ & $6,70^{* *}$ & $1,61^{\mathrm{ns}}$ & $8,21 * *$ & $2,09^{\text {ns }}$ & $7,71 * *$ & $3,61 *$ & $6,63 * *$ & $6,63^{* *}$ & $484,92 * *$ & $81,29 * *$ \\
\hline Salinidade (S) & $1,74^{\mathrm{ns}}$ & $18,03 * *$ & $5,89^{*}$ & $11,46^{* *}$ & $3,34^{\mathrm{ns}}$ & $11,90 * *$ & $0,62^{\text {ns }}$ & $23,07 * *$ & $23,07 * *$ & $11,53^{* *}$ & $12,57 * *$ \\
\hline Interação DxS & $2,18^{\text {ns }}$ & $1,71^{\mathrm{ns}}$ & $1,10^{\mathrm{ns}}$ & $1,49^{\mathrm{ns}}$ & $5,65^{* *}$ & $0,95^{\mathrm{ns}}$ & $8,94 * *$ & $3,73 *$ & $3,73 *$ & $2,94^{\mathrm{ns}}$ & $6,12 * *$ \\
\hline CV (\%) & 44,4 & 4,9 & 4,6 & 16,8 & 16,5 & 14,8 & 14,1 & 10,8 & 52,9 & 41,0 & 44,4 \\
\hline \multicolumn{12}{|c|}{ Densidade de estocagem } \\
\hline $\mathrm{D}_{20}$ & $1.496 \pm 283 b$ & $18,7 \pm 0,9 \mathrm{a}$ & $22,4 \pm 1,2$ & $50,2 \pm 9,4 \mathrm{a}$ & $103 \pm 14$ & $13,1 \pm 3,6 \mathrm{a}$ & $14,5 \pm 4,6$ & $95 \pm 5$ & $5,5 \pm 4,8$ & $34,0 \pm 1,7 \mathrm{c}$ & $3.505 \pm 506$ \\
\hline $\mathrm{D}_{40}$ & $1.753 \pm 205 \mathrm{a}$ & $18,2 \pm 0,9 \mathrm{ab}$ & $22,7 \pm 0,9$ & $44,8 \pm 5,9 \mathrm{ab}$ & $108 \pm 17$ & $10,9 \pm 2,7 \mathrm{ab}$ & $17,5 \pm 3,8$ & $90 \pm 6$ & $9,7 \pm 6,3$ & $65,0 \pm 4,5 b$ & $7.049 \pm 1.377$ \\
\hline $\mathrm{D}_{60}$ & $1.722 \pm 164 \mathrm{ab}$ & $17,8 \pm 0,6 \mathrm{~b}$ & $21,9 \pm 0,9$ & $40,9 \pm 3,9 \mathrm{~b}$ & $96 \pm 20$ & $9,2 \pm 1,9 \mathrm{~b}$ & $16,7 \pm 4,2$ & $90 \pm 9$ & $10,5 \pm 8,9$ & $96,6 \pm 9,6 \mathrm{a}$ & $9.438 \pm 2.488$ \\
\hline \multicolumn{12}{|c|}{ Salinidade da água } \\
\hline $\mathrm{S}_{0}$ & $1.565 \pm 294$ & $18,5 \pm 0,5 \mathrm{a}$ & $21,8 \pm 0,9 b$ & $45,6 \pm 6,6 \mathrm{a}$ & $102 \pm 17$ & $11,2 \pm 2,9 a$ & $16,0 \pm 4,6$ & $94 \pm 4$ & $6,5 \pm 3,9$ & $67,5 \pm 30,1 \mathrm{a}$ & $7.055 \pm 3.437$ \\
\hline $\mathrm{S}_{2}$ & $1.666 \pm 221$ & $18,8 \pm 0,8 \mathrm{a}$ & $23,2 \pm 0,6 \mathrm{a}$ & $50,6 \pm 8,3 \mathrm{a}$ & $110 \pm 8$ & $13,3 \pm 3,1 \mathrm{a}$ & $15,7 \pm 3,8$ & $96 \pm 4$ & $3,8 \pm 3,4$ & $68,4 \pm 28,1 \mathrm{a}$ & $7.592 \pm 3.270$ \\
\hline $\mathrm{S}_{4}$ & $1.739 \pm 202$ & $17,4 \pm 0,5 b$ & $22,0 \pm 1,0 \mathrm{~b}$ & $39,6 \pm 2,7 \mathrm{~b}$ & $95 \pm 22$ & $8,6 \pm 1,3 b$ & $17,1 \pm 4,2$ & $85 \pm 7$ & $15,4 \pm 7,0$ & $59,6 \pm 23,9 \mathrm{~b}$ & $5.344 \pm 1.679$ \\
\hline
\end{tabular}

${ }^{(1)}$ Médias seguidas por letras iguais, nas colunas, não diferem entre si pelo teste de Tukey, a $5 \%$ de probabilidade; $\mathrm{D}_{20}$ : 20 larvas $\mathrm{L}^{-1}$; $\mathrm{D}_{40}: 40$ larvas L ${ }^{-1} ; \mathrm{D}_{60}: 60$ larvas $\mathrm{L}^{-1} ; \mathrm{S}_{0}$ : água doce; $\mathrm{S}_{2}: 2 \%$ de salinidade; $\mathrm{S}_{4}: 4 \%$ de salinidade. (2)Para as análises estatísticas, os dados foram transformados em arc sen $(\mathrm{x} / 100)^{0,5}$. ns Não-significativo. * e **Significativo a 5 e $1 \%$ de probabilidade, respectivamente. 
Artemia sp. podem ser mantidos vivos por mais de 24 horas à salinidade de 5\%. No entanto, neste trabalho, mesmo com maior sobrevivência da Artemia sp. em $\mathrm{S}_{2}$ e $S_{4}$, a salinidade não contribuiu para reduzir os níveis de amônia não ionizada.

Aos cinco dias de alimentação, verificaram-se efeitos significativos da densidade de estocagem $(p<0,01)$ e da salinidade da água $(\mathrm{p}<0,01)$, sem efeito da interação densidade $\mathrm{x}$ salinidade, quanto ao comprimento total $\mathrm{e}$ peso das larvas de L. alexandri (Tabela 1). Os melhores resultados, para ambas as variáveis, foram registrados na menor densidade $\left(\mathrm{D}_{20}\right)$, que não diferiu de $\mathrm{D}_{40}$, e em $\mathrm{S}_{2}$ que não diferiu de $\mathrm{S}_{0}$. Estes resultados indicam a possibilidade da realização da larvicultura dessa espécie, na densidade de 40 larvas $\mathrm{L}^{-1}$ e à salinidade de $2 \%$, nos primeiros cinco dias de alimentação exógena, levando-se em consideração o crescimento. Foram verificados os menores valores nesses parâmetros, em relação à maior salinidade testada $\left(\mathrm{S}_{4}\right)$.

Aos dez dias de alimentação, verificou-se quanto ao comprimento total, somente o efeito da salinidade da água $(\mathrm{p}<0,05)$, com maior média a $2 \%$ o $\left(\mathrm{S}_{2}\right)$ (Tabela 1$)$. Este fato sugere que ocorreu melhor aproveitamento do alimento fornecido nessa salinidade, pelo aumento de tempo de vida dos náuplios de Artemia sp., em comparação à água doce. Apesar do maior tempo de vida dos náuplios, o índice maior de salinidade testado estaria acima do ideal, para melhor crescimento em comprimento dessa espécie. De acordo com Altinok \& Grizzle (2001), o crescimento de peixes de água doce pode estar relacionado a diferentes índices de salinidade, em razão da alteração do requerimento energético para a regulação iônica e osmótica. Os autores observaram que juvenis de Salmo trutta apresentaram maior crescimento, com o aumento da salinidade de 0 para $3 \%$ e o menor crescimento a $9 \%$.

Semelhantemente a este trabalho, a densidade também não influenciou o comprimento de larvas L. alexandri (López \& Sampaio, 2000) e de larvas de outros peixes carnívoros como D. labrax às densidades 50 e 200 larvas L $^{-1}$ (Hatziathanasiou et al., 2002), H. lacerdae às densidades 10 e 90 larvas $\mathrm{L}^{-1}$ (Luz \& Portella, 2005) e P. corruscans às densidades $15 \mathrm{e}$ 95 larvas L $L^{-1}$ (Campagnolo \& Nuñer, 2006).

Aos dez dias, o peso não foi afetado pela densidade de estocagem nem pela salinidade da água, porém, houve efeito significativo da interação densidade $\mathrm{x}$ salinidade $(\mathrm{p}<0,01)$ (Tabela 1). A menor média foi obtida no tratamento $\mathrm{D}_{60} \mathrm{~S}_{4}$ (Tabela 2). Este fato pode ter ocorrido pelo efeito combinado entre a maior densidade e maior salinidade da água, que podem ser consideradas altas para essa espécie. Para a maior densidade $\left(D_{60}\right)$, a maior média de peso foi registrada na $S_{2}$, que não diferiu da $\mathrm{S}_{0}$. Na $\mathrm{S}_{4}$, os animais apresentaram maior peso à $\mathrm{D}_{20}$, que não diferiu da $\mathrm{D}_{40}$. Nos demais tratamentos, foram registrados valores semelhantes de peso. Também no trabalho de Luz \& Potella (2005), o peso de larvas de H. lacerdae, criadas em baixa densidade (10 larvas $\mathrm{L}^{-1}$ ), não foi afetado pelas salinidades da água doce de 2 e $4 \%$.

Assim, com relação ao crescimento em peso e comprimento, larvas de L. alexandri podem ser criadas durante os primeiros dez dias de alimentação exógena, em água salinizada a $2 \%$, independentemente da densidade de estocagem testada, com resultados semelhantes à água doce. Bons resultados de crescimento à salinidade de $2 \%$ também foram obtidos na larvicultura de Pimelodus maculatus (Weingartner \& Zaniboni Filho, 2004).

Tabela 2. Médias ( \pm desvio-padrão) das interações entre densidades de estocagem e índices de salinidade da água das variáveis peso, taxa de crescimento específico (TCE), sobrevivência, mortalidade e biomassa total de Lophiosilurus alexandri, aos dez dias de alimentação( ${ }^{(1)}$.

\begin{tabular}{cccc}
\hline Gradiente de & \multicolumn{3}{c}{ Densidades de estocagem } \\
\cline { 2 - 4 } salinidade & $\mathrm{D}_{20}$ & $\mathrm{D}_{40}$ \\
\hline \multicolumn{4}{c}{ Peso $(\mathrm{mg})$} \\
$\mathrm{S}_{0}$ & $87,2 \pm 5,3 \mathrm{Aa}$ & $115,2 \pm 20,5 \mathrm{Aa}$ & $102,9 \pm 6,8 \mathrm{ABa}$ \\
$\mathrm{S}_{2}$ & $105,9 \pm 9,1 \mathrm{Aa}$ & $112,4 \pm 9,0 \mathrm{Aa}$ & $111,8 \pm 6,4 \mathrm{Aa}$ \\
$\mathrm{S}_{4}$ & $116,0 \pm 6,0 \mathrm{Aa}$ & $96,0 \pm 16,0 \mathrm{Aab}$ & $74,3 \pm 17,4 \mathrm{Bb}$ \\
\hline \multicolumn{4}{c}{$\mathrm{TCE}(\% \text { por dia })^{(2)}$} \\
$\mathrm{S}_{0}$ & $10,7 \pm 3,1 \mathrm{Bb}$ & $18,6 \pm 1,6 \mathrm{Aa}$ & $18,7 \pm 3,1 \mathrm{Aa}$ \\
$\mathrm{S}_{2}$ & $11,7 \pm 3,1 \mathrm{Ba}$ & $16,5 \pm 0,5 \mathrm{Aa}$ & $18,7 \pm 3,2 \mathrm{Aa}$ \\
$\mathrm{S}_{4}$ & $21,0 \pm 1,5 \mathrm{Aa}$ & $17,4 \pm 2,3 \mathrm{Aab}$ & $12,8 \pm 3,4 \mathrm{Ab}$ \\
\hline \multicolumn{4}{c}{ Sobrevivência $(\%)^{(2)}$} \\
$\mathrm{S}_{0}$ & $94,5 \pm 5,5 \mathrm{Ba}$ & $93,0 \pm 3,6 \mathrm{Aa}$ & $94,0 \pm 5,8 \mathrm{Aa}$ \\
$\mathrm{S}_{2}$ & $100 \pm 0,0 \mathrm{Aa}$ & $94,9 \pm 3,5 \mathrm{Ab}$ & $93,5 \pm 0,9 \mathrm{ABb}$ \\
$\mathrm{S}_{4}$ & $90,7 \pm 3,2 \mathrm{Ba}$ & $82,8 \pm 2,8 \mathrm{Ba}$ & $80,2 \pm 9,3 \mathrm{Ba}$ \\
\hline \multicolumn{4}{c}{ Mortalidade $(\%)^{(2)}$} \\
$\mathrm{S}_{0}$ & $5,5 \pm 5,5 \mathrm{Ba}$ & $7,0 \pm 3,6 \mathrm{Aa}$ & $5,3 \pm 5,8 \mathrm{Aa}$ \\
$\mathrm{S}_{2}$ & $0,0 \pm 0,0 \mathrm{Ab}$ & $5,1 \pm 3,5 \mathrm{Aa}$ & $6,5 \pm 0,9 \mathrm{Aa}$ \\
$\mathrm{S}_{4}$ & $9,3 \pm 3,2 \mathrm{Ba}$ & $17,2 \pm 2,8 \mathrm{Ba}$ \\
\hline \multicolumn{4}{c}{ Biomassa total $(\mathrm{mg})$} \\
$\mathrm{S}_{0}$ & $2.911 \pm 257 \mathrm{Ab}$ & $7.741 \pm 1.580 \mathrm{Aa}$ & $10.514 \pm 536 \mathrm{Aa}$ \\
$\mathrm{S}_{2}$ & $3.813 \pm 328 \mathrm{Ac}$ & $7.667 \pm 340 \mathrm{Ab}$ & $11.297 \pm 719 \mathrm{Aa}$ \\
$\mathrm{S}_{4}$ & $3.790 \pm 243 \mathrm{Aa}$ & $5.739 \pm 1.055 \mathrm{Aa}$ & $6.503 \pm 2.029 \mathrm{Ba}$ \\
\hline
\end{tabular}

(1)Médias seguidas por letras iguais, minúsculas nas linhas e maiúsculas nas colunas, não diferem entre si pelo teste de Tukey, a $5 \%$ de probabilidade; $\mathrm{D}_{20}: 20$ larvas $\mathrm{L}^{-1} ; \mathrm{D}_{40}: 40$ larvas $\mathrm{L}^{-1} ; \mathrm{D}_{60}: 60$ larvas $\mathrm{L}^{-1}$; $\mathrm{S}_{0}$ : água doce; $\mathrm{S}_{2}: 2 \%$ de salinidade; $\mathrm{S}_{4}: 4 \%$ de salinidade. ${ }^{(2)}$ Para as análises estatísticas, os dados foram transformados em $\operatorname{arc} \operatorname{sen}(\mathrm{x} / 100)^{0,5}$. 
Para a taxa de crescimento específico (TCE), aos cinco dias de alimentação, verificou-se efeito significativo da densidade $(\mathrm{p}<0,01)$ e da salinidade da água $(\mathrm{p}<0,01)$, porém, sem efeito da interação densidade $x$ salinidade $(p>0,05)$ (Tabela 1$)$. A melhor TCE foi registrada na $\mathrm{D}_{20}$, que não diferiu de $\mathrm{D}_{40}$, assim como na $S_{2}$, que não diferiu da $S_{0}$. A diminuição da TCE na maior salinidade pode estar relacionada ao maior gasto energético em processos metabólicos, como verificado em juvenis de Ictalurus punctatus e Carassius auratus, quando da elevação da salinidade de zero para 9\%o (Altinok \& Grizzle, 2004).

Entre o 6o e o 10o dia de avaliação, verificou-se efeito da densidade de estocagem $(\mathrm{p}<0,05)$ e da interação densidade $x$ salinidade $(p<0,01)$, sem efeito isolado da salinidade na TCE (Tabela 1). A criação em água doce, a 2 ou 4\% também não afetou a TCE de H. lacerdae (Luz \& Portella, 2002). Para L. alexandri, a menor TCE foi registrada no tratamento $\mathrm{D}_{20} \mathrm{~S}_{0}$ (Tabela 2). Porém, nessa mesma densidade, verificou-se maior TCE na $S_{4}$. Este resultado indica que houve melhor aclimatação dos animais a essa salinidade, com o desenvolvimento das larvas, confirmada nas demais densidades estudadas que apresentaram TCE semelhantes, entre as diferentes salinidades da água, e superiores entre o $6^{\circ}$ e $10^{\circ}$ dia de criação, em comparação aos primeiros cinco dias de trabalho. De acordo com Fashina-Bombata \& Busari (2003), a tolerância de "catfish" Heterobranchus longifilis a diferentes salinidades pode aumentar com o crescimento dos animais, o que poderia explicar esse incremento da TCE de $L$. alexandri, entre o 6o e 10 dia de alimentação. Por sua vez, uma diminuição da TCE com o aumento da densidade foi registrada a $4 \%$, o que confirma que a maior densidade e a maior salinidade testadas, em associação, são prejudiciais para essa espécie, assim como observado para os dados de peso. Esses resultados de TCE diferem dos verificados em outras espécies de larvas de peixes carnívoros, como G. morhua (Baskerville-Bridges \& Kling, 2000), D. labrax (Hatziathanasiou et al., 2002) e H. lacerdae (Luz \& Portella, 2005), que apresentaram TCE semelhantes entre as diferentes densidades estudadas. Para H. lacerdae observou-se, ainda, redução nos valores da TCE com o decorrer do desenvolvimento dos animais (Luz \& Portella, 2005).

Para a sobrevivência e a mortalidade, verificou-se o efeito da densidade $(\mathrm{p}<0,01)$, da salinidade da água $(\mathrm{p}<0,01)$ e da interação densidade $\mathrm{x}$ salinidade (Tabela 1$)$.
A sobrevivência foi maior no tratamento $\mathrm{D}_{20} \mathrm{~S}_{2}$ (Tabela 2). A salinidade de 2\%o também proporcionou melhora na sobrevivência de Clarias gariepinus, durante o desenvolvimento embrionário (Borode et al., 2002), e na larvicultura de P. maculatus (Weingartner $\&$ Zaniboni Filho, 2004), enquanto a sobrevivência de larvas de $H$. lacerdae, criadas na água doce e na salinizada a 4\%o, não foi afetada (Luz \& Portella, 2002). Para juvenis de L. alexandri, a sobrevivência foi menor a $4 \%$, independentemente da densidade utilizada, o que mostra que a espécie é mais sensível a essa salinidade do que H. lacerdae. À menor densidade de estocagem, verificou-se que a maior salinidade da água apresentou resultados de sobrevivência semelhantes à criação em água doce. Isso mostra que a maior salinidade, associada

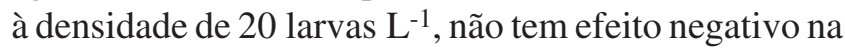
sobrevivência, ao contrário do que foi registrado em relação às maiores densidades. De acordo com FashinaBombata \& Busari (2003), a diminuição da sobrevivência com o aumento da salinidade é uma resposta característica de peixes de água doce; esses autores verificaram que exemplares de $H$. longifilis, com 28 dias de vida, apresentaram sobrevivência de $100 \%$ entre as salinidades de 0 a 6\%o, após 96 horas de observação, e diminuição na sobrevivência em salinidades superiores.

$\mathrm{O}$ aumento da densidade de estocagem, em água doce e à salinidade de $4 \%$, não afetou a sobrevivência dos juvenis de L. alexandri, ao contrário do registrado à salinidade de $2 \%$, que apresentou redução na sobrevivência com o aumento da densidade, apesar de os resultados terem sido superiores a 93\% (Tabela 2). $\mathrm{O}$ aumento de 150 para 500 larvas, por canaleta com área útil de $0,43 \mathrm{~m}^{2}$, mantidas em água doce, afetou negativamente a sobrevivência de L. alexandri (López \& Sampaio, 2000), semelhantemente ao verificado neste trabalho quanto à salinidade de $2 \%$ e contrário ao observado na água doce. No entanto, os valores de sobrevivência registrados por esses autores, 60 e $37 \%$ na menor e maior densidade, respectivamente, foram inferiores aos do presente trabalho. As divergências de resultados podem ser atribuídas aos diferentes sistemas de criação e estratégia alimentar adotados. $\mathrm{O}$ aumento da densidade de estocagem também afetou negativamente a sobrevivência de algumas espécies que praticam canibalismo, como P. maculatus (Luz \& Zaniboni Filho, 2002) e P. corruscans (Andrade et al., 2004; Campagnolo \& Nuñer, 2006), porém, esta relação não tem sido constatada em todas as espécies do mesmo hábito alimentar como G. morhua (Baskerville-Bridges 
\& Kling, 2000), D. labrax (Hatziathanasiou et al., 2002) e H. lacerdae (Luz \& Portella, 2005). Assim, não podemos concluir que exista, de maneira geral, uma relação direta entre densidade de estocagem e sobrevivência de peixes de mesmo hábito alimentar e, portanto, são necessários estudos com cada espécie, em particular.

Para a mortalidade, a menor taxa foi registrada no tratamento $\mathrm{D}_{20} \mathrm{~S}_{2}$, enquanto as maiores taxas foram verificadas nas demais densidades $\left(\mathrm{D}_{40}\right.$ e $\left.\mathrm{D}_{60}\right)$ a $4 \%$ o (Tabela 2). Porém, as larvas mortas não apresentaram sinais evidentes de agressão, e foi considerada inexistente a ocorrência de canibalismo nas condições deste trabalho. Este resultado, com os de sobrevivência e crescimento, indica o potencial da espécie para a larvicultura intensiva. Nos tratamentos $D_{40} S_{4}$ e $D_{60} S_{4}$, a mortalidade poderia estar relacionada: aos maiores níveis de amônia não ionizada na água, como relatado em relação a G. morhua (Baskerville-Bridges \& Kling, 2000), e este efeito tóxico pode ter sido potencializado pelo maior $\mathrm{pH}$ nessa salinidade (Emerson et al., 1975); à inabilidade de algumas larvas em se alimentar em altas densidades, como verificado em D. Labrax (Hatziathanasiou et al., 2002); e à menor tolerância da espécie à salinidade de $4 \%$, associada à maior densidade.

Ao contrário deste trabalho, López \& Sampaio (2000) registraram o canibalismo em L. alexandri, com o aumento da densidade de estocagem, quando as larvas foram alimentadas com zooplâncton. Possivelmente, neste trabalho, a alimentação com Artemia sp. tenha exercido importante influência para a ausência de canibalismo, uma vez que as larvas apresentaram evidencias da ingestão dos náuplios, como corpo alaranjado nos primeiros dias de alimentação, cor característica dos náuplios, e estômago repleto de náuplios, observado em animais mais velhos, o que mostra boa aceitação desse alimento. O uso de Artemia sp. também foi eficiente para evitar o canibalismo na larvicultura de $H$. lacerdae, em água salinizada (Luz \& Portella, 2002) e em diferentes densidades de estocagem (Luz \& Portella, 2005).

O número de juvenis produzidos apresentou diferenças significativas quanto à densidade $(\mathrm{p}<0,01)$ e à salinidade da água $(\mathrm{p}<0,01)$ (Tabela 1). A densidade de 60 larvas $\mathrm{L}^{-1}$ produziu maior número de indivíduos, e a salinidade de 4\%o, menor número. Esses resultados se refletiram na biomassa final, que sofreu efeito da densidade $(\mathrm{p}<0,01)$, da salinidade da água $(\mathrm{p}<0,01)$ e da interação densidade $\mathrm{x}$ salinidade $(\mathrm{p}<0,01)$. Foram observadas maiores biomassas nos tratamentos $\mathrm{D}_{60} \mathrm{~S}_{0}$ e $\mathrm{D}_{60} \mathrm{~S}_{2}$, que não diferiram entre si, e menores biomassas no tratamento $\mathrm{D}_{20}$, independentemente da salinidade da água (Tabela 2). O aumento na produção, em razão da densidade de estocagem, também foi registrado na larvicultura de P. maculatus (Luz \& Zaniboni Filho, 2002) e H. lacerdae (Luz \& Portella, 2005). Para um sistema de produção, o número final de indivíduos e a biomassa são parâmetros importantes, que indicam a relação produção e espaço. A densidade de 60 larvas $\mathrm{L}^{-1}$, em água doce ou salinizada a $2 \%$, pode ser utilizada na larvicultura do L. alexandri e otimizar o uso do laboratório para a produção de juvenis.

\section{Conclusões}

1. Larvas de Lophiosilurus alexandri podem ser criadas em água doce ou a $2 \%$ o de salinidade, em qualquer das densidades utilizadas.

2. Pela taxa de crescimento específico, as larvas de L. alexandri se mostram mais aclimatadas à maior salinidade da água após os primeiros cinco dias de alimentação.

3. Entre as densidades testadas na larvicultura de L. alexandri, as maiores densidades de estocagem, independentemente da salinidade da água, proporcionam maior número de indivíduos e biomassa final do que a menor densidade.

4. Náuplios de Artemia sp. são um ótimo recurso alimentar para larvas de L. alexandri e proporcionam elevadas taxas de sobrevivência, baixas taxas de mortalidade e ausência de canibalismo, independentemente da densidade de estocagem e da salinidade da água.

\section{Agradecimentos}

À Fundação de Amparo à Pesquisa do Estado de Minas Gerais, ao convênio entre a Companhia de Desenvolvimento dos Vales do São Francisco e do Parnaíba e a Companhia Energética de Minas Gerais, ao Prof. Dr. Nilo Bazzoli e ao Dr. Yoshimi Sato, pelo apoio financeiro, infra-estrutura e logística para a realização deste trabalho; ao Dr. Gilmar Bastos dos Santos, pelo auxílio no uso do programa estatístico.

\section{Referências}

ALTINOK, I.; GRIZZLE, J.M. Effects of brackish water on growth, feed conversion and energy absorption efficiency by juveniles euryhaline and freshwater stenohaline fishes. Journal of Fish Biology, v.59, p.1142-1152, 2001. 
ALTINOK, I.; GRIZZLE, J.M. Excretion of ammonia and urea by phylogenetically diverse fish species in low salinities. Aquaculture, v.238, p.499-507, 2004.

ANDRADE, L.S.; HAYASHI, C.; SOUZA, S.R.; SOARES, C.M. Canibalismo entre larvas de pintado, Pseudoplatystoma corruscans, cultivadas sob diferentes densidades de estocagem. Acta Scientiarum, v.26, p.299-302, 2004.

BASKERVILLE-BRIDGES, B.; KLING, L.J. Larval culture of Atlantic cod (Gadus morhua) at high stocking densities. Aquaculture, v.181, p.61-69, 2000.

BEUX, L.F.; ZANIBONI FILHO, F. Influência da baixa salinidade na sobrevivência de náuplios de Artemia sp. Boletim do Instituto de Pesca, v.31, p.73-77, 2006.

BOLASINA, S.; TAGAWA, M.; YAMASHITA, Y.; TANAKA, M. Effect of stocking density on growth, digestive enzyme activity and cortisol level in larvae and juveniles of Japanese flounder, Paralichthys olivaceus. Aquaculture, v.259, p.432-443, 2006.

BORODE, A.O.; BALOGUM, A.M.; OMOYENI, B.A. Effect of salinity on embriyonic development, hatchability, and growth of African catfish, Clarias gariepinus, eggs and larvae. Journal of Applied Aquaculture, v.12, p.89-93, 2002.

BOWER, C.E.; BIDWELL, J.P. Ionization of ammonia in seawater: effects of temperature, $\mathrm{pH}$ and salinity. Journal of the Fisheries Research Board of Canada, v.35, p.1012-1016, 1978.

CAMPAGNOLO, R.; NUÑER, A.P.O. Sobrevivência e crescimento de larvas de surubim, Pseudoplatystoma corruscans (Pisces, Pimelodidae), em diferentes densidades de estocagem. Acta Scientiarum, v.28, p.231-237, 2006.

EMERSON, K.; RUSSO, R.C.; LUND, R.E.; THURSTON, R.V. Aqueous ammonia equilibrium calculations: effects of $\mathrm{pH}$ and temperature. Journal of the Fisheries Research Board of Canada, v.32, p.2379-2383, 1975.

FASHINA-BOMBATA, H.A.; BUSARI, A.N. Influence of salinity on the developmental stages of African catfish Heterobranchus longifilis (Valenciennes, 1840). Aquaculture, v.224, p.213-222, 2003.

FEIDEN, A.; HAYASHI, C.; BOSCOLO, W.R. Desenvolvimento de larvas de surubim-do-iguaçu (Steindachneridion melanodermatum) submetidas a diferentes dietas. Revista Brasileira de Zootecnia, v.35, p.2203-2210, 2006.

GARCIA, L.O.; BECKER, A.G.; COPATTI, C.E.; BALDISSEROTTO, B.; RADÜNZ NETO, J. Salt in the food and water as a supportive therapy for Ichthyophthirius multifiliis infestation on silver catfish, Rhamdia quelen, fingerlings. Journal of the World Aquaculture Society, v.38, p.1-11, 2007.

HATZIATHANASIOU, A.; PASPATIS, M.; HOUBART, M.; KESTEMONT, P.; STEFANAKIS, S.; KENTOURI, M. Survival, growth and feeding in early life stages of European sea bass (Dicentrarchus labrax) intensively cultured under different stocking densities. Aquaculture, v.205, p.89-102, 2002.

JOBLING, M. Fish bioenergetics. London: Chapman \& Hall, 1994. 294p.

JOMORI, R.K.; CARNEIRO, D.J.; MALHEIROS, E.B.; PORTELLA, M.C. Growth and survival of pacu Piaractus mesopotamicus (Holmberg, 1887) juveniles reared in ponds or at different initial larviculture periods indoors. Aquaculture, v.221, p.277-287, 2003.

KESTEMONT, P.; JOURDAN, S.; HOUBART, M.; MÉLARD, C.; PASPATIS, M.; FONTAINE, P.; CUVIER, A.; KENTOURI, M.; BARAS, E. Size heterogeneity, cannibalism and competition in cultured predatory fish larvae: biotic and abiotic influences. Aquaculture, v.227, p.333-356, 2003.

KOROLEFF, F. Determination of nutrients. In: GRASSHOFF, K. (Ed). Methods of sea water analysis. Weinhein: Verlag Chemie, 1976. p.117-181.

LEE, C.S.; OSTROWSKI, A.C. Current status of marine finfish larviculture in the United States. Aquaculture, v.200, p.89-109, 2001.

LÓPEZ, C.M.; SAMPAIO, E.V. Sobrevivência e crescimento larval do pacamã Lophiosilurus alexandri Steindachner, 1876 (Siluriformes, Pimelodidae), em função de três densidades de estocagem em laboratório. Acta Scientiarum, v.22, p.491-494, 2000.

LUZ, R.K.; MARTÍNEZ-ÁLVAREZ, R.M.; PEDRO, N.; DELGADO, M.J. Growth, food intake regulation and metabolic adaptations in goldfish (Carassius auratus) exposed to different salinities. Aquaculture, v.276, p.171-178, 2008.

LUZ, R.K.; PORTELLA, M.C. Diferentes densidades de estocagem na larvicultura do trairão Hoplias lacerdae. Acta Scientiarum, v.27, p.95-101, 2005.

LUZ, R.K.; PORTELLA, M.C. Larvicultura de trairão (Hoplias lacerdae) em água doce e água salinizada. Revista Brasileira de Zootecnia, v.31, p.829-834, 2002. (Suplemento).

LUZ, R.K.; ZANIBONI FILHO, E. Larvicultura do mandi-amarelo Pimelodus maculatus Lacépède, 1803 (Siluriformes: Pimelodidae) em diferentes densidades de estocagem nos primeiros dias de vida. Revista Brasileira de Zootecnia, v.31, p.560-565, 2002.

LUZ, R.K.; ZANIBONI FILHO, E. Utilização de diferentes dietas na primeira alimentação do mandi-amarelo (Pimelodus maculatus, Lacépède). Acta Scientiarum, v.23, p.483-489, 2001.

SHIBATA, O.A. Family Pseudopimelodidae. In: REIS, R.E.; KULLANDER, S.O.; FERRARIS JÚNIOR, C.J. Check list of the freshwater fishes of South and Central America. Porto Alegre: Edipucrs, 2003. p.401-405.

SHIELDS, R.J. Larviculture of marine finfish in Europe. Aquaculture, v.200, p.55-88, 2001.

STATSOFT. Statistica: data analysis software system: version 7.0. Tulsa: Statsoft, 2004.

TRAVASSOS, H. Nótula sobre o pacamã. Lophiosilurus alexandri Steindachner, 1876. Atas da Sociedade de Biologia do Rio de Janeiro, v.4, p.1-2, 1959.

WEINGARTNER, M.; ZANIBONI FILHO, E. Efeito de fatores abióticos na larvicultura de pintado-amarelo Pimelodus maculatus (Lacépède, 1803): salinidade e cor de tanque. Acta Scientiarum, v.26, p.151-157, 2004.

ZANIBONI FILHO, E. Larvicultura de peixes de água doce. Informe Agropecuário, v.21, p.69-77, 2000.

$\overline{\text { Recebido em } 25 \text { de outubro de } 2007 \text { e aprovado em } 19 \text { de junho de } 2008}$ 